\title{
Directional letter-by-letter analysis and the word-superiority effect
}

\author{
GARVIN CHASTAIN \\ Boise State University, Boise, Idaho
}

\begin{abstract}
Manipulations were introduced in three experiments to produce letter-by-letter analysis of orthographic (CVC) and nonorthographic (CCV or VCC) trigrams. Letters in the trigrams were presented in a different form (normal orientation and order, normal orientation but reversed order, reversed orientation but normal order, or reversed orientation and order) to each of four groups in the first experiment, and in each of the two normal orientation forms to different groups in the second experiment. Subjects both detected the presence or absence of a target letter and classified each trigram as a word or nonword. Additional changes were introduced in the third experiment to ensure that letters were being analyzed in the desired order. Performance was consistently better on orthographic trigrams, but only if the letters were oriented normally. This word-superiority effect (WSE) was related to feature testing that may be carried out letter by letter, with more efficient testing on words. Familiar orientation of letter features seems to be necessary; otherwise, testing becomes so difficult that there is no WSE. However, testing apparently is not finished on a given letter before it is begun on the next.
\end{abstract}

A letter from a small predesignated set can be identified correctly more often if it appears in a letter string that forms a word than if it appears in a string of randomly chosen letters (Carr, Lehmkuhle, Kottas, AstorStetson, \& Arnold, 1976; Chastain, 1981; Paap \& Newsome, 1980b; Spector \& Purcell, 1977). Questions persist about the nature of the processes that result in this word-superiority effect (WSE). One issue is whether the positions in the string must be simultaneously analyzed for the WSE to appear (Adams, 1979). Johnston (1981) and Johnston and McClelland (1974) found that the advantage produced by the word context is eliminated if the position of the target is known in advance of the appearance of the string. Apparently, the other letters are not analyzed with the target, if at all. Some investigators have attempted to encourage serial analysis of the positions in the string by using wide interletter spacing. This manipulation has been found to eliminate the WSE (Purcell \& Stanovich, 1982; Purcell, Stanovich, \& Spector, 1978; but see Paap \& Newsome, 1980a), but perhaps again because it discourages analysis of all the letters presented. Finally, Travers (1975) found that serial presentation of letters in the string is more detrimental to letter identification in words than in nonwords. However, although no statistical analysis was reported, a weak WSE still seems to be present in those data. A WSE definitely appeared in a sequential presentation study reported by Krueger (1971), but since no

The author would like to thank two anonymous reviewers for their helpful suggestions on an earlier draft of this article. Requests for reprints should be addressed to Garvin Chastain, Department of Psychology, Boise State University, Boise, ID 83725 . masks were used, it is difficult to relate this to a typical WSE, which requires masking (Johnston \& McClelland, 1973). Nevertheless, serial presentation of adjacent letters introduces lateral interference that may affect letter analysis (Estes, Allmeyer, \& Reder, 1976).

Assuming a model similar to that proposed by Smith (1971), a letter string is analyzed through feature tests performed at each letter position. At no position are enough features tested to permit identification of the letter if that letter appeared in isolation, but there are sufficient tests to allow identification of a letter in a word if other features are tested at other positions. Due to orthographic constraints known to the observer, testing is more efficient in words than in nonword strings. Hence, a WSE appears. The question addressed currently is whether a WSE will be observed when such tests are conducted serially on a position-by-position basis. It would be desirable to induce serial analysis in a way that would avoid the problems accompanying the techniques used by the investigators cited earlier.

Spatially transforming letter strings has been shown to induce letter-by-letter analysis. Terry, Samuels, and LaBerge (1976) found that presenting mirror-image words produced a positive relationship between word length and response latency in a categorization task, and Lawry and LaBerge (1981) observed increases in response latency to transformed strings in a lexical decision task. Lawry and LaBerge also found that presenting mirror-image strings, strings in which the letters were normally oriented but were reversed in their order, or were normally ordered but were reversed in their orientation, each resulted in performance suggesting serial analysis. Therefore, in addition to the serial analysis issue, determination could be made of whether letters 
must be normally oriented and/or ordered for WSE to occur. Perhaps features at the various positions in a string must have a familiar orientation and order, or otherwise testing becomes so difficult that there is no WSE. Although Well, Pollatsek, and Schindler (1975) found that same-different judgments on two strings, both of which were rotated $180 \mathrm{deg}$, could be made more quickly on words, Bryden (1970) observed almost complete elimination of facilitation from familiar letter sequences when strings were displayed vertically in a full-report task. Differences in task demands (e.g., memory, report, etc.) between these studies makes comparison of their results difficult. The current experiments could provide additional evidence on the effect .on the WSE of placing letters in an unfamiliar orientation and/or order.

Thus, Experiment 1 explored the importance to the WSE of simultaneous analysis of letters in a string and of familiar letter orientation/order by displaying word (consonant-vowel-consonant, or CVC) and nonword (CCV or VCC) strings containing letters that were normally oriented and ordered (NN), normally oriented and reversed in their order (NR), reversed in their orientation and normally ordered (RN), and reversed in both orientation and order (RR), in a target search (detection) task. Lowercase letters, which were not bilaterally symmetrical and thus were physically different in their normal and reversed orientations, were used. A single lowercase letter was the target, and that letter was always $r$. In addition to reporting whether the target letter was present or absent in each string, each subject was also required to categorize the string as a word or a nonword to ensure that he or she attempted to analyze all the letters in the transformed strings serially in the desired order.

\section{EXPERIMENT 1}

\section{Method}

Subjects. Four groups of eight undergraduate subjects per group served. The subjects, each of whom reported normal or corrected-to-normal vision, were given extra course credit for their participation.

Apparatus. A Scientific Prototype Model N-1000 threechannel manual tachistoscope with a binocular viewing hood was equipped with a microswitch to allow the subjects to initiate exposures. The luminance of the stimulus and masking fields was 44.0 and $130.0 \mathrm{~cd} / \mathrm{m}^{2}$, respectively, as measured by a Spectra Lumicon with photospot attachment. A $40-\mathrm{W}$ bulb shielded from the subject dimly illuminated the laboratory.

Stimuli. Trigrams were traced in black ink from a Pilot Razor Point pen onto white index cards with an E-Z Letter plastic stencil, lowercase Gothic No. 81. Trigrams subtended a visual angle averaging $1.82 \mathrm{deg}$ horizontally. Short letters were $.64 \mathrm{deg}$ high, ascenders were $.86 \mathrm{deg}$ high, and descenders were $1.02 \mathrm{deg}$ high. The mask was a checkerboard pattern that subtended a visual angle of $1.26 \mathrm{deg}$ vertically $\times 3.36 \mathrm{deg}$ horizontally. The checks were squares $.21 \mathrm{deg}$ on a side. An $\mathrm{X}$ that subtended a visual angle of $.16 \mathrm{deg}$ in each direction was placed near the center of the pattern. Separated from the top and bottom of the pattern by $.37 \mathrm{deg}$ were arrows $.91 \mathrm{deg}$ long, centered horizontally, and both pointing either to the left or to the right.
Experimental design. Each subject received five presentations of each trigram listed in Appendix A. The orientation and order of letters in the trigrams were factorially combined to produce four sets of trigrams, each of which was shown to a different group. Four additional word trigrams (rex, sex, fan, far) and their nonword counterparts were used for practice, with the orientation and order of letters identical to the criterion trigrams for that group. The target (lowercase r) appeared as the leftmost letter on $25 \%$ of all trigrams and the rightmost letter on $25 \%$. A foil letter (n or s) appeared as the leftmost letter on an additional $25 \%$ of all trigrams and as the rightmost letter on the remaining $25 \%$. Each block of 32 criterion trials included one exposure of each trigram in Appendix A. The block of 32 practice trials included four presentations of each practice trigram. Orders were randomized within the constraints just mentioned.

Procedure. The subjects were told that they could initiate each exposure at any time after a .2-sec tone from the tachistoscope had signaled that the stimulus was in place. After each exposure, the subject said "yes" or "no" to indicate the presence or absence of the target letter, and then "word" or "nonword" to classify the trigram. The arrows on the mask, which was always illuminated except during the exposure of a trigram, served as a reminder of the direction in which the letters were to be considered in making the word/nonword classification. During the 32-trial practice block, the exposure duration was lowered from an initial setting of $200 \mathrm{msec}$ to produce an overall level of accuracy on the target detection task of between $70 \%$ and $80 \%$. Thereafter, the duration was raised or lowered only between blocks of trials when it was necessary to maintain accuracy within that range. Presentations proceeded in an uninterrupted series, and no feedback regarding accuracy was given.

\section{Results}

Exposure duration. Analysis of the mean exposure duration required by each subject showed a significant effect of letter orientation $[F(1,28)=13.58, p<.01]$. Relevant means and standard deviations, respectively, were: NN, $21 \mathrm{msec}, 7.02 \mathrm{msec}$; NR, $24 \mathrm{msec}, 5.54 \mathrm{msec}$; $\mathrm{RN}, 50 \mathrm{msec}, 30.93 \mathrm{msec}$; and RR, $55 \mathrm{msec}, 33.18 \mathrm{msec}$. A Newman-Keuls analysis showed that all means differed significantly from one another $(\mathrm{p}<.05)$ except for 21 versus $24 \mathrm{msec}$ and 50 versus $55 \mathrm{msec}$.

Target detection. Mean proportion of target detection responses correct for the two target positions in CVC and non-CVC trigrams for each subject in each group was entered into an analysis of variance, with groups analyzed as a $2 \times 2$ (orientation $\times$ order) factorial variable. The target was detected more accurately in words than in nonwords $[\mathrm{F}(1,28)=29.33, \mathrm{p}<.01]$, although context interacted with orientation $[F(1,28)=21.29$, $p<.01]$. Means for the significant main effect and interaction appear in Table 1, in which relevant means are also given for each group. Total number of errors on each trigram for each group is given in Appendix A.

The only other significant effect in the analysis of target detection accuracy was the interaction between order and target position $[F(1,28)=8.73, p<.01]$. Means appear in Table 2. A Newman-Keuls analysis revealed a significant difference only between the RN and $R R$ groups $(p<.05)$. An additional analysis of the interaction between positions for the NN and NR groups produced no significant effect $(p>.10)$.

Examination of word and nonword trigrams reveals 
Table 1

Mean Proportion of Target Detections Correct in the Significant Interaction Between Context and Orientation in Experiment 1

\begin{tabular}{lrc}
\hline \multirow{2}{*}{ Group } & \multicolumn{2}{c}{ Context } \\
\cline { 2 - 3 } NN & Word & Nonword \\
NR & .794 & .684 \\
Mean & .800 & .716 \\
RN & .797 & .700 \\
RR & .734 & .745 \\
Mean & .745 & .719 \\
Overall Mean & .740 & .732 \\
\hline
\end{tabular}

Note $-N=$ normal $; R=$ reversed .

Table 2

Mean Proportion of Target Detections Correct for Leftmost and Rightmost Target Positions in Strings Shown in Experiment 1

\begin{tabular}{lcc}
\hline & \multicolumn{2}{c}{ Target Position in Trigram } \\
\cline { 2 - 2 } Group & Leftmost & Rightmost \\
\hline NN & .764 & .717 \\
RN & .813 & .667 \\
Mean & .789 & .692 \\
NR & .748 & .772 \\
RR & .686 & .778 \\
Mean & .717 & .775 \\
\hline
\end{tabular}

Note $-N=$ normal,$R=$ reversed .

that, whereas most word trigrams have an ascender or a descender at the extreme position opposite to the target, nonword trigrams have a short letter in both extreme positions. Trigram shape thus may have aided determination of target presence in words. For the two groups showing a WSE (NN and NR), overall detection accuracy on the four word trigrams containing all short letters was compared with that on the other word trigrams. No significant difference was apparent for either group or for the combined data (all ps $>.10$ ). A marginally significant WSE appeared for these two groups even when only the exposures of trigrams containing all short letters were analyzed $[\mathrm{t}(15)=2.05, .05<\mathrm{p}<$ .10].

A final analysis of detection accuracy was performed to ensure that the differences observed were not due to response bias. It was not possible to treat the data in a signal detectability analysis because several subjects either never missed the presence of the target or never incorrectly reported an absent target as present in one of the contexts. Therefore, responses to words and nonwords were merely corrected for bias for each subject (Gummerman, 1972), and the resulting means were entered into an analysis of variance. All effects that attained significance when the raw score data were analyzed were again significant with correction for response bias, but no additional significant effect emerged.

Word-nonword classification. Proportion of responses correct on the judgment of whether each trigram exposed was a word or not for each subject in each group was entered into a one-way analysis of variance. There was no significant difference between the four groups $(\mathrm{p}>.10)$, with an overall proportion of responses correct of .771. A second analysis showed no significant difference between trigrams containing only short letters and those containing an ascender or a descender, and no significant interaction of this variable with groups (both ps $>.10$ ). In addition, since each subject made incorrect classifications of both words and nonwords, the proportion of hits (words called words) and false alarms (nonwords called words) were converted to $\mathrm{z}$ scores for signal detectability analysis. The resulting $d^{\prime}$ s for each subject in each group were entered into a one-way analysis of variance. The difference between groups was not significant $(p>.10)$, with a mean overall $d^{\prime}$ of 1.29 .

\section{Discussion}

A WSE was observed with strings containing normally oriented letters in both orders but not with the strings containing letters that were reversed in their orientation. Since reversing the order of letters has been found to induce letter-by-letter analysis (Lawry \& LaBerge, 1981), a WSE with the NR strings suggests that serial analysis of the positions in a string allows a WSE to emerge. Its absence with the $R N$ and $R R$ strings suggests that normal letter orientation is necessary to allow serial tests at the positions to proceed in a manner conducive to a WSE. The lack of a significant relationship between trigram shape and accuracy on the word/nonword judg. ment suggests that trigram shape was not being used in lieu of letter-by-letter analysis by the group receiving the NR strings. However, since a vowel in the center position always indicated that the string was a word, perhaps it was not necessary for the subjects in that group to examine the letters individually to determine whether a trigram was a word. If the vowels were more discriminable from other letters in the center position than targets were from foils, enough time may have been available for both determinations to be made on the NR strings in the way they were made on the NN strings. Reversed letter orientation may have proven to be so disruptive that it was necessary for the subjects receiving those strings to analyze all letters anyway, as indicated by exposure durations that were, on the average, over twice as long as those for the subjects receiving normally oriented letters. The longer durations were necessary to maintain overall accuracy within the desired range when letter orientation was reversed.

Although the means were in the expected direction, NR strings did not differ significantly from NN strings with respect to accuracy of target detection at the left- 
most and rightmost positions or to the exposure duration necessary for target detection accuracy in the desired range. Thus, there is no assurance that letters in the NR strings were examined individually in a right-toleft order. To provide such assurance, a second experiment, in which only half the CVCs actually were words, was run. The nonword CVCs were formed by replacing the vowel in eight of the criterion word trigrams (and two of the practice word trigrams) used in Experiment 1 with a different vowel to produce a nonword. All 16 criterion CVCs used in Experiment 2 (which appear in Appendix B) were listed in random order, and the list was given to each of 32 undergraduate subjects. Instructions were to place a check beside each trigram that formed a word. The majority judged the eight trigrams that had been altered (cen, cer, hun, hur, rem, sem, ruy, suy) to be nonwords (although hun and rem are actually words). In addition, ret was judged by the majority to be a nonword, but was retained and analyzed as a word in Experiment 2 as it had been in Experiment 1. One group in Experiment 2 received NN strings, and another received NR strings. A consistent WSE across groups in conjunction with an indication that trigrams were being analyzed letter by letter would suggest the sufficiency of serial tests on normally oriented letters for the effect.

\section{EXPERIMENT 2}

\section{Method}

Two major differences distinguish this experiment from the preceding one. Two groups of 10 undergraduate subjects per group served. Their essential characteristics were identical to those of the subjects in Experiment 1. One group received the trigrams in Appendix B with letters in NN form, and the other group received those same trigrams with letters in their NR form. Each subject received four criterion blocks of 32 trials per block.

\section{Results}

Exposure duration. Mean exposure duration did not differ between the groups $(p>.10)$. The mean for the group receiving the $\mathrm{NN}$ strings was $27.4 \mathrm{msec}$ ( $\mathrm{SD}=$ 5.98 ), and the mean for the other group was $30.1 \mathrm{msec}$ $(\mathrm{SD}=6.51)$.

Target detection. An initial analysis of proportion of target detections correct on CVCs designated as words and nonwords for both groups indicated no significant main effect of context and no significant interaction with groups (both ps $>.10$ ). All CVCs were therefore considered as words in the overall analysis of target detection accuracy. This analysis demonstrated a significant overall WSE $[F(1,18)=26.71, p<.01]$ and no interaction of the WSE with groups $(p>.10)$. Planned comparisons showed a significant difference between the proportion of responses correct on words and nonwords for the group receiving the NN strings [.803 and .741, respectively; $\mathrm{t}(9)=2.80, \mathrm{p}<.05]$ and for the group receiving the NR strings $[.858$ and .767 , respectively; $t(9)=4.66, p<.01]$. There was also a significant interaction between groups and target position $[F(1,18)=$ $7.41, p<.02]$, with superior accuracy on targets in the leftmost position for the $\mathrm{NN}$ strings (proportions correct $=.813$ and .731 for leftmost and rightmost positions, respectively) and on those in the rightmost position for the NR strings (proportions correct $=.780$ and .845 for leftmost and rightmost positions, respectively). No other main effect or interaction was significant. Total number of errors on each trigram is given in Appendix B. Analysis of the detection data corrected for response bias again showed a significant detection superiority in word context $[F(1,18)=13.37, p<.01]$ and no interaction between groups $(p>.10)$. As in Experiment 1, comparison of detection accuracy on the small subset of trials upon which trigrams containing only short letters were presented showed a marginally significant WSE $[\mathrm{t}(19)=$ $1.85, .05<\mathrm{p}<.10$ ]

Word-nonword classification. Raw data from the word/nonword judgment task were first converted to proportion of responses correct across the 96 exposures for each subject in which nonwords were presented and the remaining 32 in which words were shown. Analysis showed no reliable difference between the groups $(\mathrm{p}>.10)$, with a mean proportion correct of .690 . Data were converted to $z$ scores for signal detectability analysis and the resulting d's were compared. Again, no significant difference emerged between groups $(p>.10)$, with a mean overall $\mathrm{d}^{\prime}$ of 1.13 .

\section{Discussion}

Making some CVCs words and others nonwords and requiring a lexical decision after each exposure appears to have forced members of each group to analyze trigrams on a letter-by-letter basis, as shown by detection accuracy on targets at the two positions differing in opposite directions for the two groups. Nevertheless, a WSE was apparent for both groups. Results of Experiment 2 thus support the tentative conclusion from Experiment 1 that simultaneous analysis of letters in the strings is not necessary to yield a WSE.

However, two problems prevent the results of Experiment 2 from providing more conclusive evidence for the hypothesis under consideration. First, as in Experiment 1 , the WSE on trigrams containing only short letters was not quite statistically significant. The possibility remains that the superior overall performance on orthographic (CVC) strings was due to easier determination of which position could contain the target when only one of the two potential ones contained a short letter (such as the target $\mathrm{r}$ ). Second, although the significant interaction between groups and target position suggests that subjects receiving the NR strings were analyzing the letters in a right-to-left order, it is also possible that the interaction was due to subjects in the two groups biasing their fixation in different directions.

Therefore, a third and final experiment in which all stimuli were NR trigrams composed of block capital 
letters was run. The task required a judgment of whether each string contained a $\mathbf{P}$ or an $\mathrm{R}$. Half the trigrams began in the right visual field so that the leftmost letter was in the same position as the fixation cross; the rightmost letter in the remaining trigrams was at the position of the cross, with successive letters extending into the left visual field. A WSE was predicted, with higher accuracy when the target was the rightmost letter in the string. In addition, for targets appearing at fixation, performance when the target was the rightmost letter of trigrams extending into the left visual field was expected to be significantly better than performance when the target was the leftmost letter of trigrams beginning in the right visual field, reflecting right-to-left serial analysis of the trigrams' letters.

\section{EXPERIMENT 3}

\section{Method}

Subjects. Ten subjects, whose relevant characteristics were the same as those of subjects in the first two experiments, served.

Apparatus. The apparatus and its settings were identical to those in the preceding experiments.

Stimuli. Trigrams were prepared in the same manner as those in the preceding experiments, except that Gothic captial letters were traced from the stencil used in those experiments. The trigrams, which appear in Appendix C, subtended a visual angle of $.70 \mathrm{deg}$ vertically and, on the average, $2.4 \mathrm{deg}$ horizontally. Each trigram appeared on two slides, with its rightmost letter in the center on one slide and its leftmost letter in the center on the other. The checkerboard mask with the arrows pointing to the left that had been used in the first two experiments was extended by three checks on each side to subtend a visual angle of 4.62 deg horizontally.

Experimental design. Each of the 16 trigrams in Appendix C was presented once with its rightmost letter in the center and once with its leftmost letter in the center in each of seven blocks of trials. Presentation orders were randomized within each block, and the first block was considered to be practice.

Procedure. The procedure was that followed in the first two experiments.

\section{Results}

As in the preceding experiments, all CVCs were orthographic and were therefore analyzed as words. Analysis of variance showed that targets were better discriminated in the orthographic context $[F(1,9)=6.54$, $\mathrm{p}<.05]$, with proportions of responses correct of .786 (to the CVCs) and .749 (to the remaining trigrams). The main effect of target position was significant $[F(1,9)=9.61, p<.025]$, with .813 of targets in the rightmost position of the trigrams being correctly identified, but only .723 of those in the leftmost position. No other main effect or interaction was significant. It may be worth noting that the word advantage for the rightmost position (.045) was at least as great as that for the leftmost (.030), with no hint of a significant interaction between context and position $(F<1.0)$. Total number of errors on each trigram is given in Appendix C.

An additional analysis was done on targets appearing in the center of the screen in both orthographic and nonorthographic trigrams. Analysis of variance showed that central targets occupying the rightmost position in the string were identified significantly better than those occupying the leftmost position $[\mathrm{F}(1,9)=5.77, \mathrm{p}<.05]$, with no significant interaction between position and context.

Mean proportion of word/nonword judgments correct was .620 after correction for response bias had been applied.

\section{Discussion}

Analysis of the results of the current experiment revealed a WSE and provided assurance that serial, right-to-left, letter-by-letter analysis was occurring. Accuracy was higher on the rightmost (initial) letter than on the leftmost letter of the RN trigrams used, and there were two pieces of evidence to suggest that misfixations to the right of center did not produce the effects. First, there was no significant main effect of visual field. Second, when only targets in the center of the screen were considered, accuracy was significantly higher when the target was the initial letter of a trigram extending to the left than when it was the terminal letter of a trigram beginning to the right. Serial right-to-left analysis of the individual letters of the trigram would produce just this pattern of results, since the target in the center would be the first to be analyzed if it were the initial letter of the trigram, but the last to be analyzed if it were the terminal letter. The appearance of the mask would interfere with analysis; therefore, identification of the terminal letter would be incorrect more often than identification of the initial letter, although they would occupy the same position, if letters were being analyzed in the required order.

\section{GENERAL DISCUSSION}

A significant WSE was observed only with trigrams having letters appearing in their normal orientation and order (NN) and in their normal orientation but reversed order (NR) in Experiment 1, and again with trigrams having these same characteristics in Experiment 2 . The effect was apparently not an artifact of differences in the shape of word and nonword trigrams, since a marginally significant WSE was present with the small subset of trigrams containing only normally oriented short letters in both experiments. A significant WSE with NR trigrams was observed in Experiment 3, with an additional statistical test providing assurance that misfixations were not yielding the results and that letters were being analyzed serially in a right-to-left order.

The findings are consistent with a model that assumes that tests for features are conducted at each position in a letter string and can be performed serially on a positionby-position basis and still yield a WSE. Thus, testing tends to be more efficient with words than with nonwords. However, features at the various positions must 
have a familiar orientation for a WSE to emerge, because, otherwise, testing becomes so difficult that it is no more efficient with words than with nonwords.

Some observations reported in the literature may appear to pose problems for such an account. McClelland (1976) reported a WSE of about equal magnitude with mixed-case and same-case strings. However, Smith (1971) proposed that there is overlap in the critical features of upper- and lowercase letters and that there are functionally equivalent feature lists for different forms of the same letter. Changing feature lists may adversely affect testing, and, indeed, McClelland (1976) reported a substantial performance decrement with mixed-case strings. However, word and nonword strings were affected to approximately the same extent, preserving a WSE, and indicating that more efficient testing was still possible on the word strings.

With serial analysis of letter positions, one might expect an interaction between target position and a WSE. That is, if the initial position in the string is tested first, and at that point the subject does not know whether or not the string is orthographic, testing should be equally efficient at that position with word and nonword strings. Identification accuracy should thus be equal at the initial position, but perhaps at the terminal position differences in the efficiency of testing with the two string types would yield a WSE. A WSE is sometimes greater for targets at the terminal position (Greenberg \& Krueger, 1980), but this has not been observed consistently (Chastain, 1982; Paap \& Newsome, 1980b) and was not found in the current experiments. Apparently, even when serial, letter-by-letter analysis has occurred, differences in the way in which orthographic and nonorthographic strings are treated begin very early. Probably, analysis is not completed on a given letter before it is begun on the next (Sperling, 1967). This has led some researchers (e.g., Turvey, 1973) to suggest a mixed parallel-serial process, with overlap at different levels of analysis. An activation model such as that proposed by McClelland and Rumelhart (1981), which allows feedback from higher levels based upon activation from partial analysis at lower levels, could also account for this finding. For the current account to be correct, differences in analysis between word and nonword strings must become important before analysis of the initial letter is completed.

Finally, Lawry and LaBerge (198i) measured response latency to perform a lexical decision task on lowercase letter strings embodying each of the four combinations of orientation and order used in Experiment 1. Before each string was presenied, a priming stimulus appeared. Latency was shortest on the NN strings, but was shorter on the RR strings than on either the NR or the RN strings. The last two did not differ significantly. The authors argued that relationships between letters or letter features are preserved in the RR strings, and these may be important for word recognition. Their results appear inconsistent with those reported here, in which a WSE emerged with RN, but not with RR, strings. The apparent inconsistency serves to emphasize the difficulty of comparing results from studies involving quite different tasks, even if the studies are assumed to tap some of the same processes. It also shows that word recognition is a complex phenomenon, and that the results of experiments that approach it in very different ways are often difficult to reconcile (Gough \& Cosky, 1977).

\section{REFERENCES}

Adams, J. J. (1979). Models of word recognition. Cognitive Psychology, 11, 133-176.

Brynen, M. P. (1970). Left-right differences in tachistoscopic recognition as a function of familiarity and pattern orientation. Journal of Experimental Psychology, 84, 120-122.

Carr, T. C., Lehmkuhle, S. W., Kottas, B., Astor-Stetson, E. C., \& Arnold, D. (1976). Target position and practice in the identification of letters in varying context: A word superiority effect. Perception \& Psychophysics, 19, 412-416.

Chastain, G. (1981). Phonological and orthographic factors in the word-superiority effect. Memory \& Cognition, 9, 389-397.

Chastain, G. (1982). Scanning, holistic encoding, and the wordsuperiority effect. Memory \& Cognition, 10, 232-236.

Estes, W. K., Allmeyer, D. H., \& Reder, S. (1976). Serial position functions for letter identification at brief and extended exposure durations. Perception \& Psychophysics, 19, 1-15.

Gough, P. B., \& Cosky, M. J. (1977). One second of reading again. In N. J. Castellan, Jr., D. B. Pisoni, \& G. R. Potts (Eds.), Cognitive theory (Vol. 2). Hillsdale, NJ: Erlbaum.

Greenbera, S. N., \& Krueger, L. E. (1980). Limitations on the word-superiority effect with a fixed target set. Bulletin of the Psychonomic Society, 5, 25-28.

Gummerman, K. (1972). A response-contingent measure of proportion correct. Journal of the Acoustical Society of America, 52, 1645-1647.

Johnston, J. C. (1981). Effects of advance precuing of alternatives on the perception of letters alone and in words. Journal of Experimental Psychology: Human Perception and Performance, 7, 560-572.

Johnston, J. C., \& McClelland, J. L. (1973). Visual factors in word perception. Perception \& Psychophysics, 14, 365-370.

Johnston, J. C., \& McClelland, J. L. (1974). Perception of letters in words: Seek not and ye shall find. Science, 184, 1192-1194.

Krueger, L. E. (1971). Effect of direction of sequential presentation and redundancy on short-term recognition memory. Perception \& Psychophysics, 9, 121-124.

LAWRY, J. A., \& LABERGE, D. (1981). Letter and word code interactions elicited by normally displayed words. Perception \& Psychophysics, 30, 71-82.

McClelland, J. (1976). Preliminary letter identification in the perception of words and nonwords. Journal of Experimental Psychology: Human Perception and Performance, 2, 80-91.

MCClelland, J. L., \& RumelhaRT, D. E. (1981). An interactive activation model of context effects in letter perception: Part 1. An account of basic findings. Psychological Review, 88, 375-407.

PaAp, K. R., \& Newsome, S. L. (1980a). Do small visual angles produce a word superiority effect or differential lateral masking? Memory \& Cognition, 8, 1-14.

PAAP, K. R., \& NEWsome, S. L. (1980b). A perceptual-confusion account of the WSE in the target search paradigm. Perception \& Psychophysics, 27, 444-456.

Purcell, D. G., \& Stanovich, K. E. (1982). Some boundary conditions for a word superiority effect. Quarterly Journal of Experimental Psychology: Human Experimental Psychology, 34, 117-134. 
Purcell, D. G., Stanovich, K. E., \& Spector, A. (1978). Visual angle and the word superiority effect. Memory \& Cognition, 6, 3-8.

Rumelhart, D. E., \& Siple, P. (1974). Process of recognizing tachistoscopically presented words. Psychological Review, 81, 99-118.

Sмітн, F. (1971). Understanding reading. New York: Holt, Rinehart \& Winston.

Spector, A., \& Purcell, D. G. (1977). The word-superiority effect: A comparison between restricted and unrestricted alternative sets. Perception \& Psychophysics, 21, 323-328.

SPE RLiNG, G. (1967). Successive approximation to a model for short-term memory. Acta Psychologica, 27, 285-292.

Terry, P., Samuels, S. J., \& LaBerge, D. (1976). The effects of letter degradation and letter spacing on word recognition. Journal of Verbal Learning and Verbal Behavior, 15, 577-585.

Travers, J. R. (1975). Forced serial processing of words and letter strings: A reexamination. Perception \& Psychophysics, 18, 447-452.

Turvey, M. T. (1973). On peripheral and central processes in vision: Inferences from an information-processing analysis of masking with patterned stimuli. Psychological Review, 80, 1-52. Weli, A. D., Pollatsek, A., \& Schindler, R. M. (1975). Facilitation of both "same" and "different" judgments of letter strings by familiarity of letter sequence. Perception \& Psychophysics, 17, 511-520.

Appendix A

Total Number of Errors on Each Trigram Shown in Experiment 1

\begin{tabular}{lrrrclrrrr}
\hline & & & \multicolumn{7}{c}{ Non- } \\
CVC & NN & NR & RN & RR & CVC & NN & NR & RN & RR \\
\hline ret & 3 & 2 & 6 & 6 & rte & 6 & 6 & 5 & 2 \\
set & 5 & 12 & 9 & 9 & ste & 13 & 17 & 7 & 9 \\
rum & 7 & 3 & 7 & 7 & rmu & 11 & 4 & 5 & 12 \\
sum & 6 & 13 & 1 & 9 & smu & 9 & 15 & 8 & 13 \\
rut & 8 & 0 & 9 & 9 & rtu & 8 & 6 & 6 & 9 \\
nut & 21 & 17 & 16 & 10 ntu & 17 & 21 & 13 & 10 \\
ray & 4 & 3 & 10 & 9 & rya & 6 & 3 & 8 & 7 \\
say & 11 & 7 & 4 & 9 & sya & 16 & 19 & 6 & 12 \\
per & 6 & 6 & 9 & 9 & epr & 5 & 3 & 11 & 10 \\
pen & 6 & 11 & 12 & 7 & epn & 22 & 19 & 9 & 12 \\
car & 5 & 2 & 11 & 15 & acr & 15 & 3 & 19 & 11 \\
can & 16 & 9 & 17 & 6 & acn & 14 & 9 & 11 & 14 \\
fur & 9 & 4 & 12 & 14 & ufr & 9 & 6 & 19 & 18 \\
fun & 13 & 13 & 10 & 14 & ufn & 26 & 24 & 12 & 13 \\
her & 4 & 3 & 17 & 16 & ehr & 6 & 3 & 14 & 14 \\
hen & 6 & 23 & 20 & 14 & ehn & 19 & 24 & 10 & 14 \\
\hline
\end{tabular}

Appendix B

Total Number of Errors on Each Trigram Shown in Experiment 2

\begin{tabular}{lrrlrr}
\hline CVC & NN & NR & $\begin{array}{l}\text { Non- } \\
\text { CVC }\end{array}$ & NN & NR \\
\hline ret & 3 & 2 & rte & 1 & 2 \\
set & 6 & 4 & ste & 6 & 9 \\
rem & 2 & 2 & rme & 2 & 6 \\
sem & 8 & 3 & sme & 13 & 3 \\
rut & 2 & 0 & rtu & 5 & 2 \\
nut & 18 & 13 & ntu & 24 & 20 \\
ruy & 4 & 5 & ryu & 4 & 9 \\
suy & 10 & 6 & syu & 12 & 13 \\
per & 2 & 2 & epr & 6 & 5 \\
pen & 12 & 13 & epn & 22 & 14 \\
cer & 2 & 3 & ecr & 3 & 0 \\
cen & 15 & 6 & ecn & 17 & 10 \\
fur & 2 & 3 & ufr & 3 & 8 \\
fun & 14 & 16 & ufn & 24 & 16 \\
hur & 9 & 2 & uhr & 4 & 6 \\
hun & 17 & 11 & uhn & 20 & 26 \\
\hline
\end{tabular}

Appendix C

Total Number of Errors on Each Trigram Shown in Experiment 3

\begin{tabular}{lccc}
\hline CVC & NR & Non-CVC & NR \\
\hline POD & 18 & PDO & 32 \\
ROD & 31 & RDO & 32 \\
POG & 10 & PGO & 10 \\
ROG & 20 & RGO & 27 \\
CEP & 23 & ECP & 33 \\
CER & 33 & ECR & 38 \\
HEP & 44 & EHP & 39 \\
HER & 26 & EHR & 30 \\
\hline
\end{tabular}

(Manuscript received April 21, 1983;

revision accepted for publication February 8, 1984.) 